\title{
LVIII. The electrical conductivity and fluidity of solutions
}

\section{Richard Hosking}

To cite this article: Richard Hosking (1904) LVIII. The electrical conductivity and fluidity of solutions, Philosophical Magazine Series 6, 7:41, 469-484, DOI: 10.1080/14786440409463144

To link to this article: http://dx.doi.org/10.1080/14786440409463144

曲 Published online: 15 Apr 2009.

Submit your article to this journal \lceil

Џ Article views: 4

Q View related articles $\longleftarrow$

Citing articles: 6 View citing articles 5 
It is generally admitted that this assumption is illegitimate as an assumption, but (at any rate in the case of an infinitely rare gas) true in fact. But is Mr. Burbury prepared to charge with inconsistency all those that hold these views?

It need hardly be said that $I$ am very grateful to $M r$. Burbury for the kind interest he has always taken in my work. Criticism, in particular, is always of special value to anyone who, like myself, bas not worked at a subject for long. But in the present instance, although it is only with the greatest diffidence that I have ventured to try to maintain my position against Mr. Burbury's criticism, I cannot persuade myself that these criticisms have any true foundation.

J. H. JEANS.

LVIII. The Electrical Conductivity and Fluidity of Solutions. By Richard Hosking, 1851 Exhlibition Science Research Scholar *.

THE present paper describes experiments carried out in the Cavendish Laboratory, Cambridge, during the year 1903 and the Michaelmas Term 1902; and these experiments are the continuation of work performed in the Physical Laboratory, Melbourne, and described in the Philosophical Magazine for May $1902+$, in a joint paper by Professor Thomas R. Lyle and myself.

One of the main results of the Melbourne work was to show that both the Specific Molecular Conductivity and the Fluidity of the solutions used became zero at the same temperature, viz., $-35^{\circ} \cdot 5 \mathrm{C}$.

This result was baserl on the form of the curves representing the temperature variations of both these quantities between $100^{\circ} \mathrm{C}$. and $0^{\circ} \mathrm{C}$; ; but it is interesting to find that Kohlrausch $\ddagger$ quite independently arrived at the conclusion that in the case of dilute aqueous solutions, all conductivities would cease at practically the same temperature, viz., $-39^{\circ} \mathrm{C}$.

Another general result was that the fluidity-concentration isothermals and the conductivity-concentration isothermals all cut the axis of zero fluidity and conductivity respectively at the same point, representing a concentration of 10.74 normal. It was felt that these, and other conclusions arrived

* Communicated by Prof. J. J. Thomson, F.R.S.

$\dagger$ Phil. Mag. May, 1902, p. 487.

I Sitz. Akad. Wiss Berlin, Oct. 31st, 1901. 
at, needed further investigation, and with that object mainly in view the experiments described in the present paper were undertaken.

As solutions with high concentrations would be necessary to test one of these points, lithium chloride was chosen as the salt to be used.

The method of experimenting was practically unaltered. but there were slight variations which will be briefly indicated, The dilatometer described in the joint paper was again used for measuring the specific gravity of my solutions at all the temperatures.

\section{Viscosity.}

The glischrometer was changed back to the original form described in an earlier paper (Phil. Mag. March 1900, p. 274), which was more likely to give accurate values for viscosity; and the viscosity of my solutions was found in the way there described.

In this new glischrometer the following constants were determined, correct at $0^{\circ} \mathrm{C}$.

Corrected length of capillary tube $=5.4391 \mathrm{cms}$.

Mean radius of the capillary tube $=0.011592 \mathrm{cms}$.

Working volume of each limb $=3.8441 \mathrm{c.cms}$.

and in the formula

$$
\eta_{t}=\alpha \times \rho \times h \times \mathrm{T}-\beta \frac{\delta}{\mathrm{T}}(1+2 \gamma t)
$$

the values for $\log a$ and $\log \beta$ came out as $\overline{7} \cdot 521643$ and $\overline{2} \cdot 44902$.

\section{Resistance.}

The cell in which the electrical resistances were measured was similar to that described in the joint paper, and its capacity was determined by measuring in it the resistance of a standard solution of sulphuric acid ( 20 per cent. by weight). The value obtained for the capacity was $136^{\circ} 43 \mathrm{~cm} .^{-1}$ at $18^{\circ} \mathrm{C}$.

The modified arrangement of Kohlrausch's method *, by which a double commutator and a moving coil galvanometer are used instead of the coil and telephone, was employed to determine the resistances. Our previous arrangement was very similar, the magneto-alternator being used instead of the dry cells and one set of sectors on the commutator.

The commutator was driven by a water-motor, and could

* Plil. Trans. A. cxciv. p. 330 (1900). 
be kept rotating at constant speed by controlling the waterstupply with a long lever fixed to the tap.

The unit of resistance used throughout was the ohm, and the unit of concentration the number of gramme equivalents of salt per cubic centimetre of solution.

The conductivity of the water used for making up the solutions was found to be $4.9 \times 10^{-6}$ at $18^{\circ}$ C.

\section{General Arrangement of Apparatus.}

The bath contained the dilatometer, glischrometer, electrolytic cell, thermometer, and stirrer; and these were all held rigidly from above. It was enclosed in an iron jacket with glass windows at the front and back, and was heated from below by a large burner or small controlling flame as required.

Telescopes were used for reading the thermometer, observing the coincidence of meniscus and line in the glischrometer, reading the dilatometer as well as setting it, and for viewing the scale reflected from the mirror of the galvanometer.

The limbs of the glischrometer were connected by rubber tubing to the manometer and compressed-air cylinder through three-way taps, and each could be put in connexion with pressure or atmosphere independently.

An accuracy of 1 in 1000 was aimed at throughout, and corrections to stop-watch, manometer readings, and thermometers were carefully ascertained.

The temperature of the bath was kept constant within $\frac{1}{10}^{\circ} \mathrm{C}$. while the necessary readings were being taken, and the stirring was very efficient.

The lithium chloride was obtained pure. It was completely soluble in a mixture of ether and alcohol, and gave no residue when dissolved in water.

The solutions were not made up to any particular strength, but were obtained by diluting a stock solution. The densities of the solutions were measured in the dilatometer while the viscosities and resistances were being measured in the glischrometer and cell respectively.

\section{Results.}

With pure water in the dilatometer and glischrometer a set of readings was taken every $10^{\circ}$, from $0^{\circ} \mathrm{C}$. to $100^{\circ} \mathrm{C}$, and the values for the density thus obtained were correct within 0.01 per cent., and those for viscosity agreed within $0 \cdot 1$ per cent. with those originally obtained by me. 
Eight solutions were made up, and these will be referred to in the following pages as solutions $1,2,3, \ldots 8$.

The following table will give an idea of their composition and properties at $15^{\circ} \mathrm{C} . ;$ where density means specific gravity $15 / 4$, concentration means $\frac{\text { gm.-equivalent }}{\text { litre }}=n$, and normal solution $=42 \cdot 48$ gms. per litre.

\begin{tabular}{|c|c|c|c|c|c|c|c|}
\hline Solution. & Density. & $\begin{array}{l}\text { Concen- } \\
\text { tration. }\end{array}$ & Viscosity. & Fluidity. & Resistance. & $\begin{array}{l}\text { Sp. Mol. } \\
\text { Conduct. }\end{array}$ & $\mathrm{C} / \mathrm{F}$. \\
\hline Water... & 0.9992 & & .01143 & $87 \cdot 5$ & $27.6 \times 10^{6}$ & $92 \cdot 5$ & 1.057 \\
\hline & 0.9992 & 0.00645 & .01144 & $87 \cdot 4$ & 242700 & $8 \overline{1} \cdot 1$ & 0.997 \\
\hline & 1.0016 & $0 \cdot 1030$ & .01152 & 86.8 & 17210 & 76.9 & 0.886 \\
\hline $3 .$. & 1.0115 & 0.5203 & $\cdot 01220$ & $82 \cdot 0$ & 4000 & 656 & $0 \cdot 800$ \\
\hline & 1.0234 & 1.0125 & .01308 & $76 \cdot 5$ & 2281 & $59 \cdot 1$ & 0.772 \\
\hline & 1.0665 & 2.937 & .01718 & 58.2 & 1055 & 44.0 & 0.756 \\
\hline & $1 \cdot 1107$ & 5.02 & $\cdot 02331$ & $42 \cdot \overline{7}$ & 887 & $31 \cdot 0$ & 0718 \\
\hline $7 \ldots$ & $\mathrm{J} \cdot 1611$ & $7 \cdot 36$ & .03640 & 27.5 & 953 & $19 \cdot 6$ & 0715 \\
\hline & 1.2319 & 1071 & $\cdot 00805$ & $12 \cdot 4$ & 1440 & $8 \cdot 8$ & 0.710 \\
\hline
\end{tabular}

Viscosity and fluidity are given in absolute measure. Resistance is given in ohms $=R$, and

$$
\text { sp. mol. cond. }=\mathrm{C}=\frac{u t(1-g t)}{n \times 10^{-3} \times \mathrm{R}},
$$

where $x$ is the capacity of the cell and $(1-g t)$ is the temperature correction for the expansion of the glass.

Instead of Water we may write Solution of infinite dilution. In resistances of 1 and 2, conductivity of the water is allowed for.

The temperature of the bath, while readings were being taken, was generally within one or two tenths of a degree of the even temperatures $0^{\circ}, 10^{\circ}, 20^{\circ}$, \&c., \&c.; so that in calculating the values of the viscosity, resistance, and density at these even temperatures from their values at the actual temperatures observed, the amount of error introduced was extremely small.

It is the smoothed values, obtained in this way, which are collected in the following tables, A, B, and C, and we may take the actual observations of solution 5 as typical of all, and reproduce them here also. 
Solution 5. (Actual observations.)

Weight of solution in dilatometer (corrected) $=5.4123 \mathrm{gms}$.

\begin{tabular}{|c|c|c|c|c|c|c|c|c|}
\hline \multicolumn{2}{|c|}{ Bath. } & \multicolumn{3}{|c|}{ Glischrometer. } & \multirow{2}{*}{$\begin{array}{l}\text { Bath. } \\
\begin{array}{l}\text { Mean } \\
\text { Temp. }\end{array}\end{array}$} & \multirow{2}{*}{$\begin{array}{c}\text { Glischro- } \\
\text { meter. }\end{array}$} & \multirow{2}{*}{ 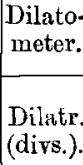 } & \multirow{2}{*}{\begin{tabular}{|c} 
Cell. \\
$\begin{array}{c}\text { Resist } \\
\text { (ohms) }\end{array}$
\end{tabular}} \\
\hline $\begin{array}{l}\text { Temp. } \\
\text { (Cent.). }\end{array}$ & $\begin{array}{l}\text { Varia- } \\
\text { tion. }\end{array}$ & $\begin{array}{r}\text { Time } \\
(\operatorname{secs} .) .\end{array}$ & $\begin{array}{c}\text { Pressure } \\
\text { (cms.). }\end{array}$ & Viscosity & & & & \\
\hline $0 \cdot 15$ & -0.00 & $556 \cdot 0$ & $143 \cdot 9$ & .02646 & $0 \cdot 15$ & .02644 & $24 \cdot 0$ & $1545 \cdot 3$ \\
\hline $0 \cdot 15$ & -0.00 & 5510 & $144 \cdot 9$ & .02642 & & & & \\
\hline $9 \cdot 70$ & $-0 \cdot 02$ & $421 \cdot 0$ & $142 \cdot 6$ & $\cdot 01984$ & $9 \cdot 71$ & .01982 & $29 \cdot 2$ & $1196 \cdot 2$ \\
\hline $9 \cdot 72$ & +0.06 & $421 \cdot 4$ & $149 \cdot 2$ & $\cdot 01980$ & & & & \\
\hline 2000 & -0.00 & $333 \cdot 1$ & $139 \cdot 2$ & 01529 & $20 \cdot 00$ & .01527 & 36.9 & $948 \cdot 8$ \\
\hline 2000 & -0.00 & $334 \cdot 1$ & $138 \cdot 5$ & 01526 & & & & \\
\hline $30-30$ & -0.00 & $302 \cdot 3$ & $122 \cdot 9$ & .0 i 222 & $30 \cdot 35$ & $\cdot 01220$ & $46 \cdot 9$ & $776 \cdot 2$ \\
\hline $30 \cdot 40$ & -0.00 & $301 \cdot 7$ & 1227 & 01217 & & & & \\
\hline 40.06 & -0.00 & $250 \cdot 0$ & $123 \cdot 4$ & 01011 & $40 \cdot 06$ & 01009 & $58 \cdot 0$ & 658.5 \\
\hline 40.06 & -000 & $249 \cdot 2$ & $123 \cdot 2$ & .01006 & & & & \\
\hline $50 \cdot 20$ & -0.00 & $219 \cdot 4$ & $118 \cdot 7$ & $\cdot 008 \overline{0} 0$ & $50 \cdot 20$ & $\cdot 00850$ & $71 \cdot 3$ & $566 \cdot 6$ \\
\hline $50 \cdot 19$ & -0.02 & $218 \cdot 6$ & $119 \cdot 0$ & .00849 & & & & \\
\hline $59 \cdot 68$ & -0.00 & $197 \cdot 2$ & $114: 2$ & 00732 & $59 \cdot 68$ & .00731 & $85 \cdot 3$ & $499-5$ \\
\hline $59 \cdot 68$ & -0.00 & $196 \cdot 4$ & 114.5 & .00731 & & & & \\
\hline $69 \cdot 98$ & +0.02 & $177 \cdot 6$ & $110^{\circ} 6$ & .00635 & $70 \cdot 02$ & .00633 & $101 \cdot 7$ & $441 \cdot 5$ \\
\hline $70 \cdot 07$ & +006 & $177 \cdot 0$ & $110 \cdot 3$ & .00631 & & & & \\
\hline $79 \cdot 92$ & $+0 \cdot 04$ & $157 \cdot 4$ & $110^{\circ} 4$ & $\cdot 00558$ & $79 \cdot 95$ & .00558 & $119 \cdot 0$ & $397 \cdot 2$ \\
\hline $79-97$ & -0.00 & $155 \cdot 8$ & $111 \cdot 5$ & -00558 & & & & \\
\hline $89 \cdot 83$ & $-0 \cdot 08$ & 1892 & $82 \cdot 0$ & .00499 & $89 \cdot 80$ & .00499 & $157 \cdot 3$ & $361 \cdot 5$ \\
\hline $89 \cdot 77$ & -0.00 & 187.4 & $82 \cdot 8$ & .00499 & & & & \\
\hline $95 \cdot 10$ & $+0 \cdot 10$ & 1776 & 82.9 & $\cdot 00472$ & $95 \cdot 15$ & .00472 & 148.4 & 3437 \\
\hline$\{95 \cdot 20$ & $+0 \cdot 10$ & $179 \cdot 8$ & $81 \cdot 9$ & $\cdot 00473$ & & & & \\
\hline
\end{tabular}

'I'ABLE A.-Density of LiCl Solutions.

\begin{tabular}{|c|c|c|c|c|c|c|c|c|c|}
\hline \multirow{2}{*}{ Temp. } & \multirow{2}{*}{ Water. } & \multicolumn{8}{|c|}{ LiCl Solutions. } \\
\hline & & 1. & 2. & 3. & 4. & 5. & 6. & 7. & 8. \\
\hline $0^{\circ} \mathrm{C}$. & 9999 & 1.0003 & $1 \cdot 0026$ & $\mid \cdot 0131$ & 1.0253 & 1.0694 & $1 \cdot 1142$ & $1 \cdot 1654$ & 1.2368 \\
\hline 10 & 9997 & 0999 & $1 \cdot 0023$ & & 1.0243 & & & & 1.2831 \\
\hline 18 & .9986 & 0.9986 & 1.0012 & 1.0110 & 1.0229 & $\bar{i} \cdot 06$ & $1 \cdot 1099$ & $1 \cdot 1601$ & $1 \cdot 2309$ \\
\hline 20 & .9983 & .9983 & 1.0009 & 1.0106 & 1.0225 & 1.0653 & $1 \cdot 1094$ & $1 \cdot 1596$ & 1.2301 \\
\hline 30 & & .9958 & 9980 & 1.0083 & 1.0197 & & & & $1 \cdot 2267$ \\
\hline 40 & .99 & .9923 & .9947 & $1.004 \tilde{5}$ & 1.0162 & 1.0590 & $1 \cdot 1032$ & $1 \cdot 1533$ & $1 \cdot 2232$ \\
\hline 50 & & .9882 & .9905 & & & & & & 1.2196 \\
\hline 60 & .9834 & .9834 & .9855 & $\cdot 9958$ & 1.0076 & 1.0509 & {$\left[\begin{array}{l}1 \\
1 \cdot 0\end{array}\right.$} & & 1.2159 \\
\hline 70 & & 977 & & .9905 & $1 \cdot 0028$ & 1.0464 & & $1 \cdot 1422$ & 1.2120 \\
\hline 80 & & 97 & .9747 & .9849 & .9972 & 1.0416 & 1.0876 & $1 \cdot 1381$ & $1 \cdot 2080$ \\
\hline 90 & & .968 & .9683 & 9790 & 9915 & 1.0366 & 1.08 & $1 \cdot 1340$ & 1.2039 \\
\hline 100 & .9586 & .9586 & .9614 & .9728 & .9856 & 1.0312 & 1.0781 & $1 \cdot 1298$ & $1 \cdot 1998$ \\
\hline
\end{tabular}


Table B.--Viscosity of LiCl Solutions.

\begin{tabular}{|c|c|c|c|c|c|c|c|c|c|}
\hline \multirow{2}{*}{ Temp. } & \multirow{2}{*}{ Water. } & \multicolumn{8}{|c|}{ LiCl Solutions. } \\
\hline & & 1. & 2. & 3. & 4. & 5. & 6. & 7. & 8. \\
\hline $0^{\circ} \mathrm{C}$ & 01794 & 01802 & .01806 & .01894 & .02025 & $\cdot 02652$ & .03558 & $\cdot 05576$ & -1290 \\
\hline 10 & .01309 & .01310 & .01320 & .01397 & $\cdot 01497$ & 01965 & 02652 & .04150 & .0937 \\
\hline 18 & .01060 & .01061 & .01070 & 01133 & .01212 & 01600 & .02169 & .03365 & .0739 \\
\hline 20 & .01009 & 01012 & .01020 & .01075 & .01154 & .01527 & .02070 & 03204 & .0699 \\
\hline 30 & .00802 & 00804 & .00810 & 00859 & 00920 & .01228 & .01664 & .02562 & $\cdot 0540$ \\
\hline 40 & .00659 & .00659 & .00668 & .00706 & .00758 & .01012 & 01366 & $\cdot 02112$ & .0431 \\
\hline 50 & .00554 & .00554 & 00561 & 00595 & 00636 & .00853 & 01156 & .01751 & .03552 \\
\hline 60 & .00472 & .00472 & .00479 & .00510 & .00542 & 00728 & .00995 & 01482 & .02957 \\
\hline 70 & .00408 & .00408 & 00414 & 00441 & .00474 & .00632 & .00865 & 01289 & -02508 \\
\hline 80 & .00358 & 00358 & 00363 & .00890 & 00417 & .00558 & .00764 & $\cdot 01133$ & $\cdot 02161$ \\
\hline 90 & .00319 & .00319 & 00323 & .00346 & 00368 & 00496 & .00686 & .01003 & .01885 \\
\hline 100 & 00287 & .00287 & |00292 & $\cdot 00308$ & 00325 & $\cdot 00444$ & .00617 & 00895 & 01660 \\
\hline
\end{tabular}

TABLE C.-Resistance of LiCl Solutions.

\begin{tabular}{|c|c|c|c|c|c|c|c|c|}
\hline \multirow{8}{*}{ Temp. } & \multicolumn{7}{|c|}{ LiCl Solutions. } \\
\cline { 2 - 9 } & 1. & 2. & 3. & 4. & 5. & 6. & 7. & 8. \\
\hline $0^{\circ} \mathrm{C}$ & 370500 & 25870 & 5974 & 3360 & 1552 & 1291 & 1398 & 2240 \\
10 & 275200 & 19460 & 4513 & 2568 & 1186 & 995 & 1070 & 1652 \\
18 & 227300 & 16050 & 3740 & 2131 & 990 & 830 & 891 & 1336 \\
20 & 217700 & 15350 & 3588 & 2033 & 949 & $796 \cdot 9$ & $853 \cdot 4$ & $1 \cdot 274$ \\
30 & 177200 & 12520 & 2931 & 1661 & $781 \cdot 1$ & $655 \cdot 8$ & $699 \cdot 5$ & 1006 \\
40 & 147600 & 10590 & 2462 & 1402 & $659 \cdot 0$ & $552 \cdot 7$ & $585 \cdot 2$ & 819 \\
50 & 126100 & 8985 & 2112 & 1205 & $568 \cdot 1$ & $476 \cdot 9$ & $500 \cdot 3$ & 680 \\
60 & 109200 & 7810 & 1840 & 1052 & $496 \cdot 5$ & $417 \cdot 5$ & $434 \cdot 8$ & 578 \\
70 & 96500 & 6910 & 1629 & $931 \cdot 3$ & $441 \cdot 5$ & $369 \cdot 4$ & $384 \cdot 0$ & 500 \\
80 & 86040 & 6190 & 1460 & $836 \cdot 6$ & $396 \cdot 9$ & $331 \cdot 3$ & $342 \cdot 3$ & 436 \\
90 & 77980 & 5585 & 1323 & 759 & 360 & $301 \cdot 5$ & $308 \cdot 9$ & 386 \\
100 & 71080 & 5080 & 1210 & 693 & 330 & $278 \cdot 0$ & $281 \cdot 5$ & 348 \\
& & & & & & & & \\
\hline
\end{tabular}

Knowing the molecular concentrations of our solutions at any one temperature, say $18^{\circ} \mathrm{C}$, we can calculate them for the other temperatures from the alteration in the density as we heat or cool the solutions.

Professor Kohlrausch kindly supplied me with the figures connecting the density of lithium chloride solutions at $18^{\circ} \mathrm{C}$. $(18 / 4)$ with the concentration; and these were used in determining the concentration of my solutions at $18^{\circ} \mathrm{C}$., and, by means of Table $A$, the concentration at the other temperatures as well. These concentrations, in grammeequivalents per litre, are given in Table $\mathrm{D}$.

Table $\mathrm{E}$ gives the fluidity values obtained by inverting the values in Table $B$; and Table $F$, by using the values for $R$ in Table C, and for $n$ in Table D, gives the values for the 
TABLE D.-Concentration ( $n$ ) of LiCl Solutions.

\begin{tabular}{|c|c|c|c|c|c|c|c|c|}
\hline \multirow{2}{*}{ Temp. } & \multicolumn{8}{|c|}{ LiCl Solutions. } \\
\hline & 1. & 2. & 3. & 4. & 5. & 6. & 7. & 8. \\
\hline $0^{\circ} \mathrm{C}$ & 00646 & $0 \cdot 1032$ & 05211 & 1.0144 & 2.945 & 504 & 7.38 & $10 \cdot 75$ \\
\hline 10 & $-006+6$ & $0 \cdot 1031$ & 0.507 & 1.0134 & 2.940 & $5 \cdot 03$ & $7 \cdot 37$ & $10 \cdot 72$ \\
\hline 18 & .00645 & $0 \cdot 1030$ & 0.5200 & 1.0120 & 2935 & 5.02 & $7 \cdot 35$ & $10 \cdot 70$ \\
\hline 20 & .00645 & $0 \cdot 1030$ & 0.5198 & 1.0116 & $2 \cdot 934$ & 502 & $7 \cdot 35$ & 1070 \\
\hline 30 & $\cdot 00643$ & $0 \cdot 1027$ & 05186 & 1.0088 & 2.925 & 500 & $7 \cdot 33$ & $10 \cdot 67$ \\
\hline 40 & $.000 i 41$ & $0 \cdot 1023$ & 0.5167 & 1.0054 & $2 \cdot 916$ & 499 & 7.31 & 1063 \\
\hline 50 & .00638 & $0 \cdot 1019$ & $0.51+5$ & $1 \cdot 0013$ & $2 \cdot 906$ & 4.97 & $7 \cdot 29$ & $10 \cdot 60$ \\
\hline 60 & $\cdot 00635$ & $0 \cdot 1014$ & 05122 & (1.4968 & $2 \cdot 894$ & 496 & $7 \cdot 26$ & 10.57 \\
\hline 70 & .06632 & $0 \cdot 1008$ & 0.5095 & 0.9921 & $2 \cdot 882$ & 4.94 & $7 \cdot 24$ & $10 \cdot 53$ \\
\hline 80 & .00628 & 0.1002 & 05066 & 0.9866 & $2.86 \%$ & 4.02 & $7 \cdot 21$ & 10.50 \\
\hline 90 & $\cdot 00624$ & 00996 & $0 \div 03 \tilde{z}$ & (1.9810 & $2 \cdot 854$ & \pm .90 & $7 \cdot 19$ & $10 \cdot 46$ \\
\hline 100 & .00619 & 00989 & 0.5004 & 0.9750 & 2.810 & 488 & $7 \cdot 16$ & 1043 \\
\hline
\end{tabular}

Table E - Fluidity (F) of LiCl Solutions.

\begin{tabular}{|c|c|c|c|c|c|c|c|c|c|}
\hline \multirow{2}{*}{ Temp. } & \multirow{2}{*}{ Water. } & \multicolumn{8}{|c|}{ LiCl Solutions. } \\
\hline & & 1. & 2. & 3. & t. & 5. & 6. & 7. & 8. \\
\hline $0^{\circ} \mathrm{C}$ & 5.75 & $55 \div 50$ & $55 \cdot 37$ & 5280 & +9.38 & $37 \cdot 71$ & $28 \cdot 10$ & $\begin{array}{l}0.93 \\
\end{array}$ & $7 \cdot 75$ \\
\hline 10 & & 7633 & & & 605 & & $37.7 \mathrm{I}$ & 24 & 1067 \\
\hline 18 & 1 & $94 \div 25$ & $93 \cdot 40$ & $88 * 26$ & $82 \cdot 50$ & 62.50 & t5 89 & 2972 & $13 \cdot 5$ \\
\hline 20 & $94 \cdot 11$ & 98.81 & 98.00 & $93 \cdot 02$ & $86 \cdot 65$ & $66 \cdot 49$ & $48 \div 31$ & 31.21 & $14 \cdot 30$ \\
\hline 30 & $124 \cdot 7$ & $124 \cdot 4$ & $123 \cdot 4$ & $116 \cdot 4$ & 108.9 & $81 \cdot 43$ & $60 \cdot 09$ & $39 \cdot 03$ & 185 \\
\hline 40 & 1517 & 151.7 & $1+9.7$ & $141 \%$ & 131.9 & $98 \cdot 84$ & 7321 & $47 \cdot 31$ & 20.300 \\
\hline 50 & & $180^{\circ}$ & & & & $117 \cdot 2$ & $86 \cdot 50$ & $57 \cdot 11$ & $28 \cdot 15$ \\
\hline 60 & $1 \cdot 4$ & 211.9 & 208 & 1960 & 184.3 & $137 \cdot 4$ & 100 & $67 \cdot 32$ & 33.82 \\
\hline 70 & & $245 \cdot 1$ & & & & 158.2 & 115 & & \\
\hline & & & 2755 & $256 \cdot 4$ & $240 \cdot 0$ & 179.2 & 1309 & $8 \times \div 26$ & 46.28 \\
\hline 90 & $313: 5$ & $313 \cdot 5$ & $309 \cdot 6$ & \pm 89.0 & 271.8 & $201 \cdot 6$ & $145 \cdot 8$ & 9070 & 53.05 \\
\hline 100 & 3484 & $348 \cdot 1$ & $3+3 \cdot 0$ & 3246 & 3077 & $.225 \cdot 2$ & $162 \cdot 0$ & 111.70 & $60 \cdot 24$ \\
\hline
\end{tabular}

Table F.-Sp. Mol. Cond. (C) of LiCl Solutions.

\begin{tabular}{|c|c|c|c|c|c|c|c|c|c|}
\hline \multirow{2}{*}{ Temp. } & \multirow{2}{*}{$\begin{array}{c}\text { Infinite } \\
\text { Dilution. }\end{array}$} & \multicolumn{8}{|c|}{ LiCl Solutions. } \\
\hline & & 1. & 2. & 3. & 4. & 5. & 6. & 7. & 8. \\
\hline $0^{\circ} \mathrm{C}$ & $60 \cdot 60$ & $57 \cdot 0$ & $51 \cdot 13$ & 4382 & 40.03 & $29 \cdot 85$ & $21 \cdot 23$ & $13 \cdot 2 \cdot 2$ & $5 \cdot 66$ \\
\hline 10 & $81 \cdot 4$ & $76 \cdot 8$ & 67.99 & $58 \cdot 0 \overline{3}$ & $52+2$ & $39 \cdot 12$ & $27 \cdot 60$ & $17 \cdot 31$ & $7 \cdot 70$ \\
\hline 18 & 98.9 & $93 \cdot 1$ & $82 \cdot 52$ & $70 \cdot 14$ & 6326 & 4695 & $33 \cdot 15$ & 2084 & $9 \cdot 54$ \\
\hline 20 & $103 \cdot 4$ & $97 \cdot 2$ & $86 \cdot 31$ & $73 \cdot 15$ & 66.33 & $49 \cdot 00$ & $34 \cdot 53$ & $21 \cdot 77$ & $10 \cdot 01$ \\
\hline 30 & $127 \cdot 6$ & $119 \cdot \overline{8}$ & $106 \cdot 1$ & $89 \cdot 75$ & $81 \cdot 41$ & $59 \cdot 70$ & $42 \cdot 08$ & $26 \cdot 61$ & 1272 \\
\hline 40 & 1534 & $144 \cdot 2$ & 1259 & $107 \cdot 2$ & 9678 & $70 \cdot 98$ & $50 \cdot 1$ & 31.9 & $15 \cdot 67$ \\
\hline 50 & 180.0 & 1695 & 149.0 & 1255 & $113 \cdot 1$ & 8264 & $58 \cdot 2$ & $37 \cdot 5$ & $18: 87$ \\
\hline 60 & $209 \cdot 6$ & $196 \%$ & $172 \cdot 3$ & $144 \cdot 7$ & $130 \cdot 1$ & $94 \cdot 9$ & 668 & $43 \cdot 3$ & $22 \cdot 33$ \\
\hline 70 & $239 \cdot 2$ & 2236 & 1957 & $164 \cdot 4$ & 1477 & $107 \cdot 3$ & $75 \cdot 7$ & $49 \cdot 2$ & 259 \\
\hline 80 & 269.6 & $25 \% 6$ & $219 \cdot 8$ & $184 \cdot 5$ & 1653 & $119 \cdot 8$ & $84 \cdot 8$ & $55 \cdot 3$ & $29 \cdot 8$ \\
\hline 90 & $299 \cdot 7$ & $280 \cdot 4$ & $\because 45 \cdot 2$ & $204 \cdot 8$ & $183 \cdot 2$ & 132.7 & 936 & 61.5 & $33 \cdot 6$ \\
\hline 100 & 3306 & $309 \cdot 8$ & $271 \% 5$ & 2253 & $201 \%$ & $145 \cdot 6$ & $100 \cdot 0$ & 67.7 & $37 \cdot 6$ \\
\hline
\end{tabular}


TABLE G.-Ratio $\frac{\mathrm{C}}{\mathrm{F}}$ for LiOl Solutions.

\begin{tabular}{|c|c|c|c|c|c|c|c|c|c|}
\hline \multirow{2}{*}{ Temp. } & \multirow{2}{*}{$\begin{array}{l}\text { Infinite } \\
\text { Dilution. }\end{array}$} & \multicolumn{8}{|c|}{ IiCl Solutions. } \\
\hline & & 1. & 2. & 3. & 4. & 5. & 6. & 7. & 8. \\
\hline $0^{\circ} \mathrm{C}$ & 1.087 & 1.027 & .923 & $\cdot 830$ & $.81 !$ & 791 & .756 & $\cdot 739$ & 734 \\
\hline 10 & $\begin{array}{l}1.066 \\
10\end{array}$ & 1.006 & .897 & $\cdot 811$ & .784 & .768 & 732 & .720 & 711 \\
\hline 18 & 1.048 & .988 & $\cdot 883$ & 795 & $\cdot 767$ & 751 & $\cdot 716$ & 703 & 703 \\
\hline 20 & 1.044 & .983 & $\cdot 881$ & $\cdot 786$ & 766 & 748 & 715 & 699 & .700 \\
\hline 30 & 1.023 & 962 & 860 & 771 & 749 & 733 & 703 & 684 & .687 \\
\hline 40 & 1.011 & .950 & .841 & .757 & 734 & 718 & 684 & .676 & -675 \\
\hline 50 & .998 & .937 & -831 & $\cdot 747$ & 719 & 704 & 673 & .657 & .670 \\
\hline 60 & .989 & .928 & .825 & 738 & 706 & .691 & $\cdot 664$ & 642 & $\cdot 660$ \\
\hline 70 & .976 & .915 & .810 & 725 & 700 & 678 & 655 & .634 & .650 \\
\hline 80 & .965 & .904 & 798 & 719 & .690 & .669 & 648 & 628 & .644 \\
\hline 90 & .956 & .895 & .792 & 709 & $\cdot 675$ & 658 & -646 & 618 & .633 \\
\hline 100 & .948 & .888 & 791 & 695 & 658 & 646 & 638 & 608 & $\cdot 624$ \\
\hline
\end{tabular}

specific molecular conductivity, in units, already mentioned. Table $G$ gives the ralues for the ratio $\overline{\mathrm{F}}$.

It will be necessary to explain how the figures in the first columns of Tables $F$ and $G$ are obtained, and for this purpose we must examine closely the other figures in Table $G$. It will be seen that for each solution the decrease in the value $\frac{\mathrm{C}}{\mathrm{F}}$ is the same for equal rises in temperature: thus between $0^{\circ}$ and $10^{\circ}$ we have decreases of $\cdot 022$ throughout, and between $80^{\circ}$ and $90^{\circ}$ decreases of .008 as an average.

If we assume that for a solution of infinite dilution the same values apply, and if we have values of the fluidity of such a solution, and the absolute value for the conductivity at any one temperature, we can calculate the values for $\frac{\mathrm{C}}{\mathrm{F}}$ at all temperatures. Now Kohlrausch has determined the value for $\mathrm{C}_{\infty}$ at $18^{\circ} \mathrm{C}$, namely $98^{\circ} 9$, and the fluidities will be those of water, so that having $\underset{\mathrm{F}}{\mathrm{C}}$ at $18^{\circ} \mathrm{C}$. equal to $1 \cdot 048$, we can build up the first column by adding or subtracting the proper differences or those which exist in solution 1-which amounts to practically the same thing.

We can now fill up the first column in Table F, for we have both values of $\frac{\mathrm{C}}{\mathrm{F}}$ and of $\mathrm{F}$ for a solution of infinite dilution, so that we know the values of $\mathbf{C}_{\infty}$ at all temperatures between $0^{\circ}$ and $100^{\circ} \mathrm{C}$.

Experiments were made on two solutions in which the 
temperatures were carried down below $0^{\circ} \mathrm{C}$., and the figures for these solutions, which we shall call numbers 9 and 10 , are here given. It will be seen that the lowest temperature reached was $-15^{\circ} \cdot 20 \mathrm{C}$.

Solution 9.

\begin{tabular}{|c|c|c|c|c|c|c|c|c|}
\hline Temp & $\begin{array}{l}\text { Varia- } \\
\text { tion. }\end{array}$ & Density. & $\begin{array}{l}\text { Concen- } \\
\text { tration. }\end{array}$ & Viscosity & Fluidity & $\begin{array}{l}\text { Resis- } \\
\text { tance. }\end{array}$ & $\begin{array}{l}\text { Sp. Mol. } \\
\text { Condd. }\end{array}$ & $\mathrm{C} / \mathrm{F}$. \\
\hline $15 \cdot 20$ & -0.00 & $1 \cdot 0500$ & $2 \div 266$ & $\cdot 01530$ & $65 \cdot 36$ & 1197 & $50: 30$ & 768 \\
\hline 0.20 & -0.00 & 1.0517 & $2 \cdot 270$ & .02346 & $42 \cdot 62$ & 1733 & $34 \cdot 68$ & 811 \\
\hline$-8 \cdot 00$ & $-0 \cdot 00$ & 10527 & $2 \cdot 272$ & .03125 & $32 \cdot 00$ & 2217 & $\because 08$ & $8+7$ \\
\hline
\end{tabular}

Solution 10.

\begin{tabular}{|r|c|c|c|c|c|c|c|c|}
\hline Terop. & $\begin{array}{c}\text { Varia- } \\
\text { tion. }\end{array}$ & Density. & $\begin{array}{l}\text { Coneen- } \\
\text { tration. }\end{array}$ & Viscosity & Fluidity. & $\begin{array}{c}\text { Resis- } \\
\text { tance. }\end{array}$ & $\begin{array}{c}\text { Sp. Mol. } \\
\text { Cond. }\end{array}$ & C/F. \\
\hline $15 \cdot 60$ & $-0 \cdot 00$ & $1 \cdot 1530$ & $6 \cdot 74$ & 03.50 & $30 \cdot 71$ & $933 \cdot 2$ & $21 \cdot 69$ & 706 \\
$0 \cdot 40$ & $+0 \cdot 04$ & $1 \cdot 1570$ & $6 \cdot 76$ & 04913 & $20 \cdot 35$ & 1346 & $14 \cdot 99$ & $\cdot 737$ \\
$-7 \cdot 15$ & $-0 \cdot 00$ & $1 \cdot 1584$ & $6 \cdot 76$ & 06293 & $15 \cdot 89$ & 1676 & $12 \cdot 04$ & 758 \\
$-12 \cdot 00$ & $+0 \cdot 04$ & $1 \cdot 1595$ & $6 \cdot 77$ & 07441 & $13 \cdot 44$ & 1964 & $10 \cdot 26$ & 763 \\
-1450 & $-0 \cdot 00$ & $1 \cdot 1599$ & 678 & 08125 & $12 \cdot 06$ & 2154 & $9 \cdot 34$ & 759 \\
$-15 \cdot 20$ & $+0 \cdot 20$ & $1 \cdot 1601$ & 6779 & 0837 & $11 \cdot 95$ & & & \\
\hline
\end{tabular}

In these experiments, the bath was of methylated spirits surrounded by a freezing mixture of crushed ice and salt, and the bath was kept weil stirred in the ordinary way.

\section{Discussion of the Results.}

Let us consider the temperature variations of fluidity and conductivity, as represented by our ten solntions, or eleven if we include the solution of infinite dilution.

It will be seen at once that the fluidity increases more rapidly than the conductivity, the former increasing its value sixfold, and the latter fivefold, in being heated through $100^{\circ}$ from $0^{\circ} \mathrm{C}$., and this applies whether the solutions be weak or dilute.

If we represent the results algebraically, we find that both the fluidity and conductivity values can be expressed by equations of the same form, and that they are proportional to the $m$ th power of the temperature measured from a fixed point below the $0^{\circ} \mathrm{C}$, which is the same for all solutions. 
This temperature is calculated to be $-48^{\circ} \cdot 10 \mathrm{C}$. Thus for the solutions in Table $\mathrm{E}$ we have the equation $\mathrm{F}=\frac{\mathrm{T}^{m}}{a}$, where $m$ and $a$ are different constants for the different solutions, and $\mathrm{T}$ is the temperature measured from $-48^{\circ} 10 \mathrm{C}$.

For the solutions in Table F, we have similar equations, as $C=\frac{T^{n}}{b}$; and of course in Table $G$, the temperature variations are represented by $\frac{\mathrm{C}}{\mathrm{F}}=\frac{a / b}{\mathrm{~T}^{m-i}}$.

The values for the constants $a, b, m, n, a / b, m-n$, have been calculated, and are collected in the following table.

The formula here used is that known as Slotte's, and was found by Thorpe and Rodger to represent better than any other the results of their experiments on viscosity.

Values of Constants.

\begin{tabular}{|c|c|c|c|c|c|c|}
\hline Solution. & $m$. & $n$ & $m-n$. & $a$. & $b$. & $a / b$. \\
\hline 8 & $1 \cdot 8631$ & $1 \cdot 7016$ & $0 \cdot 1615$ & $182 \cdot 00$ & $130 \cdot 13$ & $1 \cdot 3986$ \\
\hline 7 & $1 \cdot 6456$ & 1.4841 & $0 \cdot 1615$ & 33.290 & 24.050 & $1 \cdot 3841$ \\
\hline 6 & 15853 & 1.4256 & $0 \cdot 1597$ & $16 \cdot 650$ & $11 \cdot 847$ & 1.4054 \\
\hline 5 & 1.5933 & $1 \cdot 4306$ & $0 \cdot 1627$ & $12 \cdot 723$ & 8.555 & $1-4873$ \\
\hline 4 & $1 \cdot 6325$ & $1-4579$ & 0.1756 & $11 \cdot 392$ & $7 \cdot 091$ & $1 \cdot 6065$ \\
\hline 3 & ]$\cdot 6230$ & 1.4763 & $0 \cdot 1467$ & $10 \cdot 163$ & $6 \cdot 950$ & $1 \cdot 4620$ \\
\hline 2 & $1 \cdot 6357$ & 1.4960 & 0.1397 & $10 \cdot 165$ & $6 \cdot 403$ & 1.5874 \\
\hline$\overline{1}$ & $1 \cdot 6500$ & 1.5257 & $0 \cdot 1243$ & $10 \cdot 705$ & 6.442 & $1 \cdot 6665$ \\
\hline Inf. Dilution. & $1 \cdot 6440$ & $1-5291$ & $0 \cdot 114 y$ & 10416 & 6.145 & $1 \cdot 69.50$ \\
\hline
\end{tabular}

The following table, which gives the values for $\frac{C}{\overline{\mathrm{F}}}$ calculated

\begin{tabular}{|c|c|c|c|c|c|c|c|c|c|}
\hline \multirow{2}{*}{ Temp } & \multirow{2}{*}{$\begin{array}{l}\text { Infinite } \\
\text { Dilution. }\end{array}$} & \multicolumn{8}{|c|}{ LiCl Solutions. } \\
\hline & & 1. & 2. & 3. & 4. & 5. & 6. & 7. & 8. \\
\hline $0^{\circ} \mathrm{C}$ & $1 \cdot 086$ & $1 \cdot 025$ & 926 & 829 & 814 & 792 & 757 & 741 & 748 \\
\hline 10 & 1.063 & $1 \cdot 002$ & 990 & 806 & 787 & 770 & 735 & 718 & .796 \\
\hline 18 & $1 \cdot 048$ & 990 & 884 & ·791 & 770 & 752 & 720 & 703 & . \\
\hline 20 & 1.043 & 982 & 881 & 787 & 766 & 748 & 716 & 700 & 707 \\
\hline 30 & 1.027 & •964 & 864 & 772 & 748 & 732 & 701 & 685 & 692 \\
\hline 40 & 1013 & $\cdot 951$ & 849 & .758 & 732 & 717 & 687 & 672 & 678 \\
\hline 50 & $1 \cdot 601$ & -939 & 837 & 746 & 718 & 705 & 676 & 660 & 667 \\
\hline 60 & 989 & 927 & 8.5 & 736 & 706 & 694 & 665 & 650 & 657 \\
\hline 70 & .979 & .018 & 815 & 726 & 695 & 684 & 656 & 640 & 647 \\
\hline 80 & 970 & $\cdot 909$ & 806 & 718 & 685 & 675 & 648 & 632 & 639 \\
\hline 100 & .962 & .890 & 798 & 710 & $\cdot 668$ & 667 & .640 & 625 & 631 \\
\hline & & & & & & & & & \\
\hline
\end{tabular}


from the formulæ by using the above constants, may be compared with Table $G$, the observed values for $\underset{\mathrm{F}}{\mathrm{C}}$, and the calculated values for $G$ and $F$ represent the observed values with the same degree of accuracy.

If we treat these resuits graphically, as we have done in figures 1 and 2, where the ordinates represent temperatures

Fig. 1.

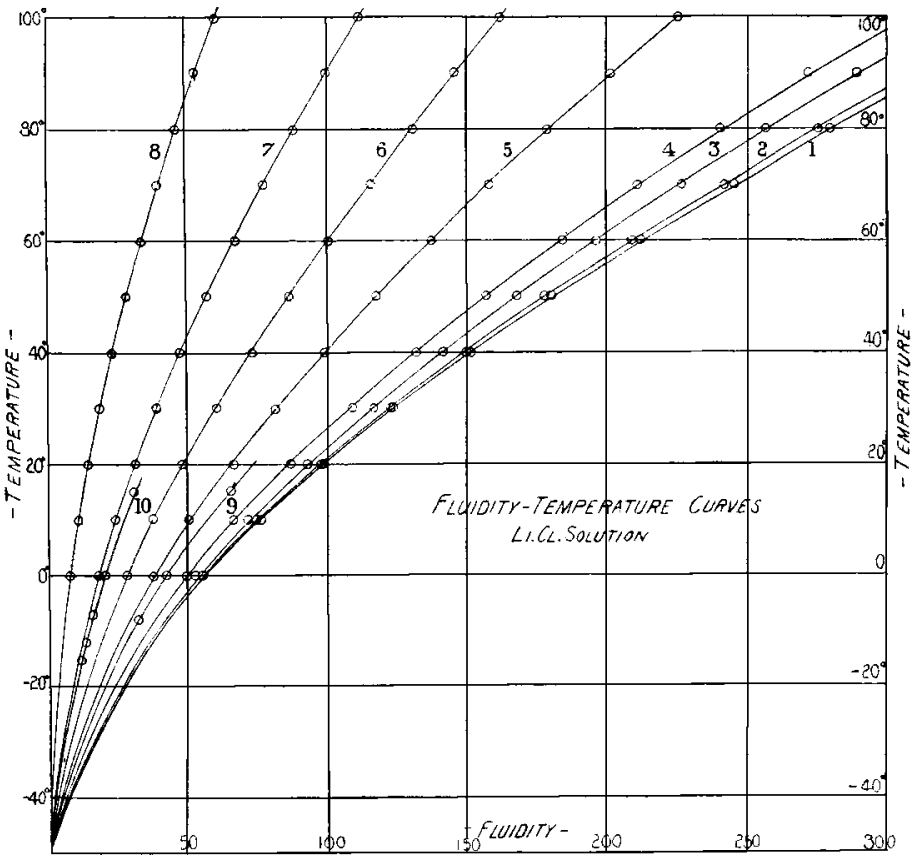

and the abscissæ the fluidity and conductivity respectively, we see that here, too, from the form of the curves above the points representing the lowest temperatures reached by experiment, the curves if continued all cut the axis representing zero fluidity and zero conductivity respectively at this same temperature of $-48^{\circ} \cdot 10 \mathrm{C}$.

On examining the figures previously obtained for sodiumchloride solutions, it was found that they could be represented by the same form of equation, which contains this temperature $-48^{\circ} .10 \mathrm{C}$. as the starting point, and it is very probable that low temperature experiments would lead to this temperature, and not $-35^{\circ} .5 \mathrm{C}$., being fixed at the point at which, apart 
from solidification, the fluidity and the conductivity of these solutions would have zero value.

Fig. 2.

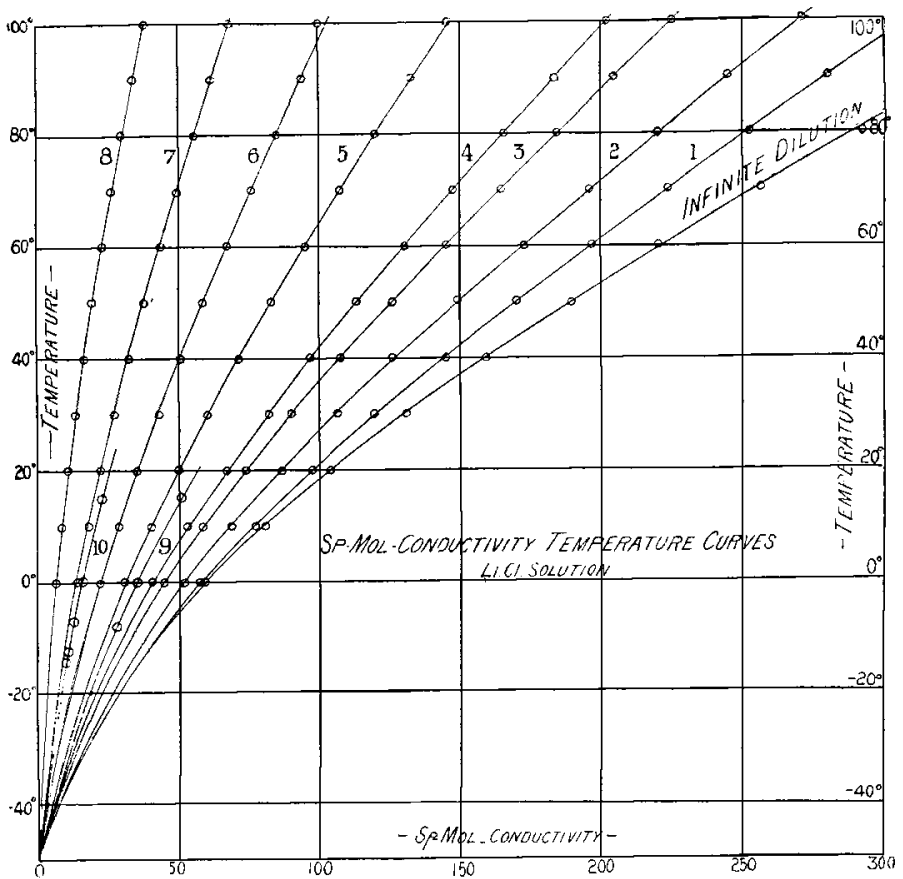

Let us calculate out the values of the ratio $\frac{\mathrm{C}_{n}}{\mathrm{C}_{a}}$ in Table $\mathrm{F}$. For the weaker solutions the numbers will measure the ionization coefficients, and we can see how these alter with change of temperature.

Ionization Coefficients.

\begin{tabular}{|c|c|c|c|c|c|c|}
\hline Temperature. & 1. & 2. & 3. & 4. & b. & 6. \\
\hline $0^{\circ} \mathrm{C}$. & .40 & $8+4$ & 723 & $\cdot 661$ & 493 & 350 \\
\hline 10. & $\cdot 943$ & 835 & 713 & $\cdot 644$ & $\cdot 481$ & 339 \\
\hline …....... & 941 & .834 & 709 & $6+0$ & +75 & $\cdot 335$ \\
\hline 20 & .940 & $\cdot 835$ & 708 & 641 & $4 \overline{i t}$ & $\cdot 334$ \\
\hline 30 & .939 & 832 & 705 & 638 & +68 & $\cdot 330$ \\
\hline 40 & 940 & 821 & 699 & (i31 & -463 & $\cdot 327$ \\
\hline 50 & .942 & 828 & 697 & 628 & 460 & 323 \\
\hline 60 & .938 & .822 & 690 & 621 & -453 & $\cdot 319$ \\
\hline 70 & 935 & 818 & 687 & $\cdot 618$ & $\cdot+49$ & $\cdot 316$ \\
\hline ....... & .937 & -815 & $\cdot 684$ & 613 & -444 & :314 \\
\hline 90 & .936 & .818 & 683 & 610 & $\cdot 443$ & 312 \\
\hline 100 & 937 & -821 & 682 & $\cdot 611$ & $\cdot 440$ & 302 \\
\hline
\end{tabular}


In solution 1 , the ionization remains constant at all temperatures, but for the other solutions there is a very marked decrease with rise of temperature, and this becomes greater the stronger the solution.

Referring back to Table $\mathbf{E}$, it will be seen that for solution 1 there has been no change in fluidity by the addition of the salt to the water. It is only when the fluidity becomes smaller by the addition of the salt that there is the diminishing of the ratio $\frac{\mathrm{C}_{n}}{\mathrm{C}_{a}}$ with rise of temperature, so that the diminution of $\frac{\mathrm{C}_{n}}{\mathrm{C}_{a}}$ may be due to two causes, the re-combination of the ions and the retarding influence of the molar viscosity; and the latter influence may be felt in much weaker solutions than one has generally supposed.

$I$ have at the begininng of this paper briefly referred to the work of Koblrausch in connexion with the zero conductivity of dilute solutions. His conclusions were arrived at from a study of the temperature variations of conductivity of dilute solutions between $0^{\circ}$ and $34^{\circ} \mathrm{C}$. The question of a lower limit to the conductivity has since been attacked by Bousfield and Lowry*, who show that the conductivity of dilute solutions and the viscosity of water tend towards the same limiting temperature, and over the range of temperature (from $5^{\circ}$ to $34^{\circ} \mathrm{C}$.) the temperature variations can be expressed by the same kind of curve. They, however, doubt the existence of the zero at the point indicated by Professor Kohlrausch and Professor Lyle and myself.

Neither Kohlrausch nor Bousfield and Lowry used Slotte's form of equation to represent their results, and it has been shown by Thorpe and Rodger $t$ that this is the one which gives the best values, where a wide range of temperature is involved, for viscosity ; and it is probably the best form of equation to use in connexion with conductivity results.

Kunz $\ddagger$ conducted some low-temperature experiments with strong sulphuric-acid solutions and solutions of other substances, and decided that in these cases no zero conductivity existed at the temperature supposed.

Kohlransch $\S$ has recently studied the temperature variations of ionic mobilities, and has here introduced the idea that ions in solutions are surrounded by watery atmospheres carried along with them, and the resistance the ions

* Bousfield \& Lowry, Roy. Soc. Proc. p. 42, June 19, 1902.

+ Thorpe \& Rodger, Phil. Trans. 1894.

$\ddagger$ Kunz, Compt. Rend. vol. exxxv. p. 788 (1902).

$\$$ Kohlrausch, Sitz. Ber d. Berlin. Akad. p. 572 (1902).

Phil. Mag. S. 6. Vol. 7. No. 41. May $1904 . \quad 2 \mathrm{~L}$ 
have to overcome is mainly friction between this atmosphere and the solvent water.

In a later paper, Kohlrausch* has explained more fully his previous paper, and has sketched out the new view of the mechanics of electrolysis, according to which the moving ion carries with it a mass of adhering solvent, and the electrical resistance of an ion is a frictional resistance which increases with the dimensions of the atmosphere surrounding it.

One of the conclusions he arrives at is that the resistance of an ion expressed in mechanical units must be of the same order of magnitude as the mechanical resistance of a molecule of the solvent.

The velocity of the ions will depend on the viscosity of the medium through which they pass, and on the size of the ionic atmosphere ; and the conductivity of the solution will depend on the viscosity of the medium, the size of the ionic atmosphere, and the fraction of dissociated ions in solution.

The lithium ion, which moves very slowly, may be considered as the centre of a larger atmosphere than the fastly moving chlorine ion, and probably with rise of temperature the atmospheres will approach the same size, as Kohlrausch has observed that with rise of temperature the velocity of the ions tend to become equal.

If we examine our weakest solution, we shall see that here the conductivity does not keep pace with the molar fluidity as the temperature is raised, although there is no combination of the ions. It looks as if the atmosphere around the neyative ion is increasing rapidly, while that around the positive ion remains constant or diminishes slowly.

$\overline{\mathrm{F}}=1.048$ at $18^{\circ} \mathrm{C}$, and consider the atmosphere of the $\mathrm{Cl}$ ion to have unit radius, that of the $L i$ ion will have $\sqrt{2}$ times this radius at the temperature $18^{\circ} \mathrm{C}$, because the $\mathrm{Cl}$ ion moves twice as fast, and the retardation will depend on the square of the radius.

Now $\frac{\mathrm{C}}{\mathrm{F}}$ at $100^{\circ}=946$. The ratio has decreased in the proportion $1 \cdot 048: \cdot 946$ or $1 \cdot 11$ to 1 . This decrease is due to the increase of the atmosphere of the ions, and as these atmospheres tend to become equal, if wo assume that at $100^{\circ}$ they are equal, the radius of each will be $1 \cdot 29$, so that the Cl has increased its atmosphere by $\cdot 29$, and the $\mathrm{Li}$ has diminished its by $\cdot 12$.

* Kohlrausch, Roy. Soc. Proc. Feb. 17, 1903. 
In solution 1, there is no combination of the ions as the temperature rises, so that here, too, any change iv the value of $\frac{\mathrm{C}}{\mathrm{F}}$ will indicate a change in the ionic atmospheres. Here also the decrease is from $1 \cdot 11$ to 1 . For this solution, $\mathrm{F}$ has the same values as for the solution just considered, but $\mathrm{C}$ has smaller values throughout because there are fewer carriers of electricity, although the atmospheres work out to have the same values as in the other solution throughour.

Solution 2 has the same increase for the radii of the atmospheres if the ionization coefficients in our table have the physical meaning which that name should imply, but here the atmospleres at any particular temperature are smaller by one per cent. If, however, the atmospheres remain of constant radius for all solutions, at any particular temperature, the figures under 2 in the table of ionization coefficients must be increased by 1 per cent. throughout to have their physical sneaning.

Fig. 3.

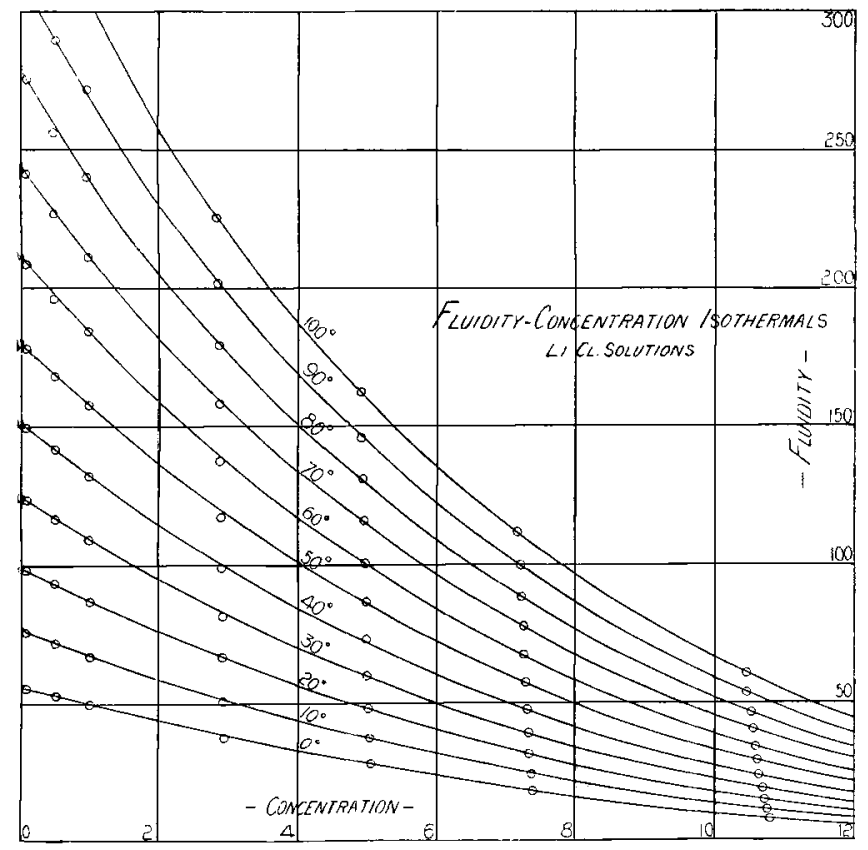

For the stronger solutions the ratio 1.11 to 1 remains throughout, bat there is nothing to indicate how the ionic atmospheres vary with the concentration. 
In the present paper I have confined myself to the temperature variations of fluidity and conductivity. The fluidityconcentration and conductivity-concentration isothermals are also interesting, but $I$ shall not discuss them here. There are indications, however, that all the isothermals will cut the axis of zero conductivity and fluidity at the same point representing concentrations of about 16 normal. I intend making further experiments on strong solutions to test this point.

Fig. 4.

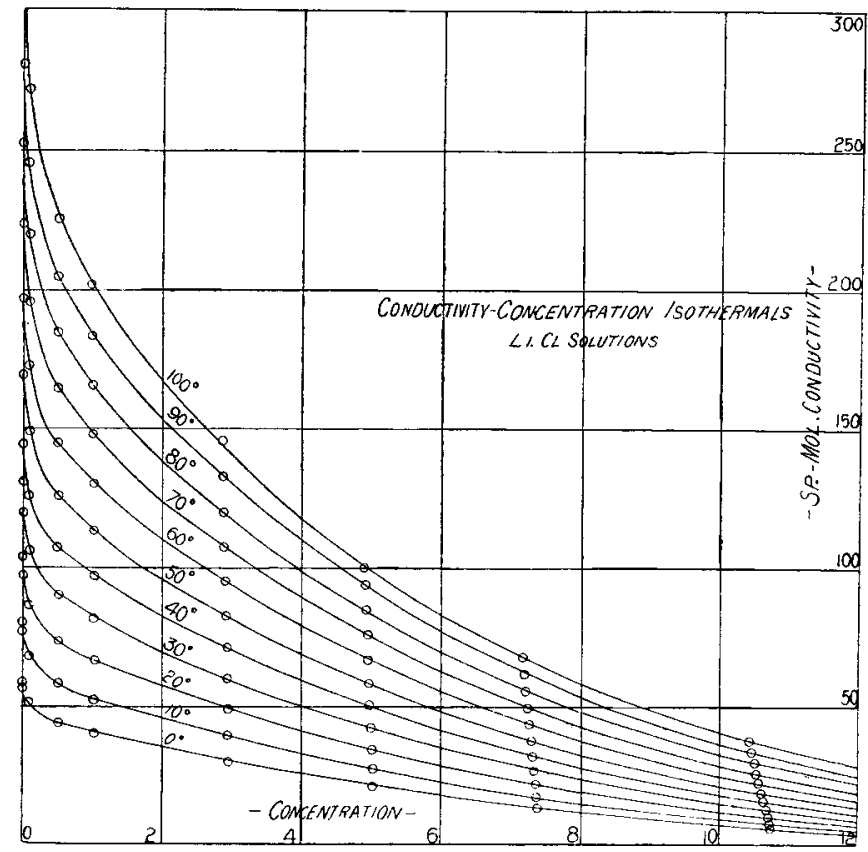

The curves connecting the variables $\mathrm{F}$ and $n$, and $\mathrm{C}$ and $n$, are given in figs. 3 and 4 respectively, each curve being the isothermal for the temperature indicated on it.

I wish, in conclusion, to thank Professor Thomson for the interest he has taken in this investigation.

Cavendish Laboratory, March 14, 1904. 